\title{
Quaternionic factorization of the Schroedinger operator and its applications to some first order systems of mathematical physics
}

\author{
Viktor G. Kravchenko*, Vladislav V. Kravchenko** \\ *Faculdade de Ciencias y Tecnologia \\ Universidade do Algarve, Campus de Gambelas \\ 8000 Faro, PORTUGAL \\ e-mail: vkravch@ualg.pt \\ **Depto. de Telecomunicaciones \\ SEPI ESIME Zacatenco \\ Instituto Politécnico Nacional \\ Av. IPN S/N, Edif. 1 \\ C.P. 07738, D.F., MEXICO \\ vkravchenko@ipn.mx
}

November 10, 2018

\section{Introduction}

We consider the following first order systems of mathematical physics.

1. The Dirac equation with scalar potential.

2. The Dirac equation with electric potential.

3. The Dirac equation with pseudoscalar potential.

4. The system describing non-linear force free magnetic fields or Beltrami fields with nonconstant proportionality factor.

5. The Maxwell equations for slowly changing media.

6. The static Maxwell system. 
We show that all this variety of first order systems reduces to the equation

$$
D f+f \cdot \vec{\alpha}=0,
$$

where $D$ is the Moisil-Theodoresco operator (introduced by Hamilton) acting on biquaternion valued functions $f$ according to the rule $D f=\sum_{k=1}^{3} e_{k} \partial_{k} f$, $\partial_{k}=\frac{\partial}{\partial x_{k}}, e_{k}$ are standard quaternionic imaginary units; the function $f$ of real variables $x_{1}, x_{2}, x_{3}$ has the form $f=\sum_{k=0}^{3} f_{k} e_{k}, f_{k} \in C, k=0,1,2,3$ and $\vec{\alpha}$ is a purely vectorial biquaternion valued function. We reduce the solution of equation (11) to the solution of a Schrödinger equation with biquaternionic potential. In some important situations the biquaternionic potential can be diagonalized and converted into scalar potentials.

\section{Notations}

We will consider the algebra $\mathbb{H}(\mathbb{C})$ of complex quaternions or biquaternions which have the form $q=\sum_{k=0}^{3} q_{k} e_{k}$ where $\left\{q_{k}\right\} \subset \mathbb{C}, e_{0}$ is the unit and $\left\{e_{k} \mid \quad k=1,2,3\right\}$ are the quaternionic imaginary units, that is the standard basis elements possessing the following properties:

$$
\begin{gathered}
e_{0}^{2}=e_{0}=-e_{k}^{2} ; e_{0} e_{k}=e_{k} e_{0}=e_{k}, \quad k=1,2,3 \\
e_{1} e_{2}=-e_{2} e_{1}=e_{3} ; e_{2} e_{3}=-e_{3} e_{2}=e_{1} ; e_{3} e_{1}=-e_{1} e_{3}=e_{2} .
\end{gathered}
$$

We denote the imaginary unit in $\mathbb{C}$ by $i$ as usual. By definition $i$ commutes with $e_{k}, k=0,1,2,3$.

The vectorial representation of a complex quaternion will be used. Namely, each complex quaternion $q$ is a sum of a scalar $q_{0}$ and of a vector $\vec{q}$ :

$$
q=\operatorname{Sc}(q)+\operatorname{Vec}(q)=q_{0}+\vec{q},
$$

where $\vec{q}=\sum_{k=1}^{3} q_{k} e_{k}$. The purely vectorial complex quaternions $(\operatorname{Sc}(q)=$ $0)$ are identified with vectors from $\mathbb{C}^{3}$. The following conjugation operations will be needed. The quaternionic conjugation is defined as follows $\bar{q}=q_{0}-\vec{q}$, the complex conjugation: $q^{*}=\operatorname{Re} q-i \operatorname{Im} q$ and the following involutive operation $q^{(k)}=e_{k} q \overline{e_{k}}$ which changes signs of two components, for example, $q^{(1)}=-e_{1} q e_{1}=q_{0} e_{0}+q_{1} e_{1}-q_{2} e_{2}-q_{3} e_{3}$.

By $M^{p}$ we denote the operator of multiplication by a complex quaternion $p$ from the right-hand side: $M^{p} q=q \cdot p$.

We will intensively use the fact that the algebra of complex quaternions contains a subset of zero divisors $\mathfrak{S}$ which are characterized by the equality 
$q_{0}^{2}=\vec{q}^{2}$, where $\vec{q}^{2}=-\langle\vec{q}, \vec{q}\rangle$, or equivalently $q^{2}=2 q_{0} q$. Hence if $q \in \mathfrak{S}$ and $q_{0}=1 / 2$ then $q$ is an idempotent. Using this fact we introduce the following multiplication operators $P_{k}^{ \pm}=\frac{1}{2} M^{\left(1 \pm i e_{k}\right)}, k=1,2,3$. It is easy to see that for each $k$ the operators $P_{k}^{+}$and $P_{k}^{-}$represent a pair of mutually complementary, orthogonal projection operators on the set of $\mathbb{H}(\mathbb{C})$-valued functions. More information on the structure of the algebra of complex quaternions can be found for example in [17] or [19].

Let $f$ be a complex quaternion valued differentiable function of $\mathbf{x}=$ $\left(x_{1}, x_{2}, x_{3}\right)$. Denote

$$
D f=\sum_{k=1}^{3} e_{k} \frac{\partial}{\partial x_{k}} f .
$$

This expression can be rewritten in vector form as follows

$$
D f=-\operatorname{div} \vec{f}+\operatorname{grad} f_{0}+\operatorname{rot} \vec{f} .
$$

That is, $\operatorname{Sc}(D f)=-\operatorname{div} \vec{f}$ and $\operatorname{Vec}(D f)=\operatorname{grad} f_{0}+\operatorname{rot} \vec{f}$. Let us notice that $D^{2}=-\Delta$. The operator $D+M^{\vec{\alpha}}$ we will denote also by $D_{\vec{\alpha}}$.

Let us introduce an auxiliary notation $\widetilde{f}:=f\left(x_{1}, x_{2},-x_{3}\right)$. The domain $\widetilde{\Omega}$ is assumed to be obtained from the domain $\Omega \subset \mathbb{R}^{3}$ by the reflection $x_{3} \rightarrow-x_{3}$.

\section{First order systems reducing to equation (1)}

\subsection{The Dirac equation with scalar potential}

Let $\mathcal{D}$ denote the classic Dirac operator for a free particle with a specified energy $\omega \in \mathbb{R}$

$$
\mathcal{D}=i \omega \gamma_{0}+\sum_{k=1}^{3} \gamma_{k} \partial_{k}+i m
$$

Here $\gamma_{j}, j=0,1,2,3$ are usual $\gamma$-matrices (see, e.g., [5]) and $m \in \mathbb{R}$.

The Dirac operator with scalar potential has the form (see, e.g., 23])

$$
\mathcal{D}^{s c}=\mathcal{D}+i \varphi_{s c} I
$$

where $\varphi_{s c}$ is a scalar real-valued function of $\mathbf{x}$ and $I$ denotes the identity operator.

In 13. (see also 17, 19]) the following transformation was introduced. A function $\Phi: \Omega \subset \mathbb{R}^{3} \rightarrow \mathbb{C}^{4}$ is transformed into a function $F: \widetilde{\Omega} \subset \mathbb{R}^{3} \rightarrow$ 
$\mathbb{H}(\mathbb{C})$ by the rule

$F=\mathcal{A}[\Phi]:=\frac{1}{2}\left(-\left(\widetilde{\Phi}_{1}-\widetilde{\Phi}_{2}\right) e_{0}+i\left(\widetilde{\Phi}_{0}-\widetilde{\Phi}_{3}\right) e_{1}-\left(\widetilde{\Phi}_{0}+\widetilde{\Phi}_{3}\right) e_{2}+i\left(\widetilde{\Phi}_{1}+\widetilde{\Phi}_{2}\right) e_{3}\right)$.

The inverse transformation $\mathcal{A}^{-1}$ is defined as follows

$$
\Phi=\mathcal{A}^{-1}[F]=\left(-i \widetilde{F}_{1}-\widetilde{F}_{2},-\widetilde{F}_{0}-i \widetilde{F}_{3}, \widetilde{F}_{0}-i \widetilde{F}_{3}, i \widetilde{F}_{1}-\widetilde{F}_{2}\right) .
$$

Let us present the introduced transformations in a more explicit matrix form which relates the components of a $\mathbb{C}^{4}$-valued function $\Phi$ with the components of an $\mathbb{H}(\mathbb{C})$-valued function $F$ :

$$
F=\mathcal{A}[\Phi]=\frac{1}{2}\left(\begin{array}{rrrr}
0 & -1 & 1 & 0 \\
i & 0 & 0 & -i \\
-1 & 0 & 0 & -1 \\
0 & i & i & 0
\end{array}\right)\left(\begin{array}{c}
\widetilde{\Phi}_{0} \\
\widetilde{\Phi}_{1} \\
\widetilde{\Phi}_{2} \\
\widetilde{\Phi}_{3}
\end{array}\right)
$$

and

$$
\Phi=\mathcal{A}^{-1}[F]=\left(\begin{array}{rrrr}
0 & -i & -1 & 0 \\
-1 & 0 & 0 & -i \\
1 & 0 & 0 & -i \\
0 & i & -1 & 0
\end{array}\right)\left(\begin{array}{c}
\widetilde{F}_{0} \\
\widetilde{F}_{1} \\
\widetilde{F}_{2} \\
\widetilde{F}_{3}
\end{array}\right)
$$

The following equality is valid

$$
D_{\vec{\alpha}_{s c}}=-\mathcal{A} \gamma_{1} \gamma_{2} \gamma_{3} \mathcal{D}^{s c} \mathcal{A}^{-1}
$$

where $D_{\vec{\alpha}_{s c}}=D+M^{\vec{\alpha}_{s c}}$ and $\vec{\alpha}_{s c}=-\left(i \omega e_{1}+\left(m+\widetilde{\varphi}_{s c}\right) e_{2}\right)$. Thus the function $\Phi$ is a solution of the Dirac equation with scalar potential

$$
\mathcal{D}^{s c} \Phi=0 \quad \text { in } \quad \Omega
$$

if and only if the function $F=\mathcal{A} \Phi$ is a solution of the equation

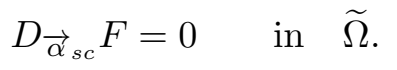

\subsection{The Dirac equation with electric potential}

The Dirac equation with electric potential has the form

$$
\mathcal{D}^{e l}=\mathcal{D}+i \varphi_{e l} \gamma_{0}
$$


where $\varphi_{e l}$ is a real-valued function.

We have an equality similar to (2):

$$
D_{\vec{\alpha}_{e l}}=-\mathcal{A} \gamma_{1} \gamma_{2} \gamma_{3} \mathcal{D}^{e l} \mathcal{A}^{-1}
$$

where $D_{\vec{\alpha}_{e l}}=D+M^{\vec{\alpha}_{e l}}$ and $\vec{\alpha}_{e l}=-\left(i\left(\omega+\varphi_{e l}\right) e_{1}+m e_{2}\right)$. Thus the equation $\mathcal{D}^{e l} \Phi=0$ is equivalent to the equation $D_{\vec{\alpha}_{e l}} F=0$.

\subsection{The Dirac equation with pseudoscalar potential}

The Dirac equation with pseudoscalar potential has the form (see, e.g., [23])

$$
\mathcal{D}^{p s}=\mathcal{D}+\varphi_{p s} \gamma_{0} \gamma_{5},
$$

where $\varphi_{p s}$ is a real-valued function. We have the following equality (see [17]), similar to (2) and (3):

$$
D+\nu I+M^{\vec{\beta}}=-\mathcal{A} \gamma_{1} \gamma_{2} \gamma_{3} \mathcal{D}^{p s} \mathcal{A}^{-1},
$$

where $\nu=-i \widetilde{\varphi}_{p s}$ and $\vec{\beta}=-\left(i \omega e_{1}+m e_{2}\right)$.

Suppose that $\vec{\beta} \notin \mathfrak{S}$, that is $m^{2} \neq \omega^{2}$ and denote $S^{ \pm}=\frac{1}{2 \lambda} M^{(\lambda \pm \vec{\beta})}$, where the complex number $\lambda$ is chosen such that $\lambda^{2}=\vec{\beta}^{2}$. We have [14]

$$
D+\nu I+M^{\vec{\beta}}=S^{+}(D+(\nu+\lambda) I)+S^{-}(D+(\nu-\lambda) I) .
$$

The operators of multiplication $S^{+}$and $S^{-}$are mutually complementary projection operators on the set of $\mathbb{H}(\mathbb{C})$-valued functions and commute with the operators in parentheses in (4). Thus $f$ satisfies the equation

$$
\left(D+\nu I+M^{\vec{\beta}}\right) f=0
$$

if and only if the functions $f^{+}=S^{+} f$ and $f^{-}=S^{-} f$ are solutions of the equations

$$
(D+\nu+\lambda) f^{+}=0
$$

and

$$
(D+\nu-\lambda) f^{-}=0
$$

respectively. In other words, given $f^{+}$and $f^{-}$solutions of (6) and (7), the function $f=S^{+} f^{+}+S^{-} f^{-}$will be a solution of (5). 
Equations (6) and (7) have a quite convenient form for studying, nevertheless in order to reduce them to the universal form (11) we make one additional step. We have

$$
D+(\nu+\lambda) I=P_{1}^{+}\left(D+M^{(\nu+\lambda) i e_{1}}\right)+P_{1}^{-}\left(D-M^{(\nu+\lambda) i e_{1}}\right)
$$

and

$$
D+(\nu-\lambda) I=P_{1}^{+}\left(D+M^{(\nu-\lambda) i e_{1}}\right)+P_{1}^{-}\left(D-M^{(\nu-\lambda) i e_{1}}\right) .
$$

Thus $f^{+}$and $f^{-}$are solutions of (6) and (7) respectively if and only if the functions $f^{++}=P_{1}^{+} f^{+}$and $f^{-+}=P_{1}^{-} f^{+}$are solutions of the equations

$$
\begin{aligned}
& \left(D+M^{(\nu+\lambda) i e_{1}}\right) f^{++}=0, \\
& \left(D-M^{(\nu+\lambda) i e_{1}}\right) f^{-+}=0,
\end{aligned}
$$

and the functions $f^{+-}=P_{1}^{+} f^{-}$and $f^{--}=P_{1}^{-} f^{-}$are solutions of the equations

$$
\begin{aligned}
& \left(D+M^{(\nu-\lambda) i e_{1}}\right) f^{+-}=0, \\
& \left(D-M^{(\nu-\lambda) i e_{1}}\right) f^{--}=0 .
\end{aligned}
$$

The obtained result we resume in the following statement.

Proposition 1 For $\vec{\beta} \notin \mathfrak{S}$ and $\lambda^{2}=\vec{\beta}^{2}$ the following equality is valid

$$
\begin{aligned}
D+\nu I+M^{\vec{\beta}} & =P_{1}^{+} S^{+}\left(D+M^{(\nu+\lambda) i e_{1}}\right)+P_{1}^{-} S^{+}\left(D-M^{(\nu+\lambda) i e_{1}}\right) \\
& +P_{1}^{+} S^{-}\left(D+M^{(\nu-\lambda) i e_{1}}\right)+P_{1}^{-} S^{-}\left(D-M^{(\nu-\lambda) i e_{1}}\right)
\end{aligned}
$$

which implies

$$
\begin{aligned}
\operatorname{ker}\left(D+\nu I+M^{\vec{\beta}}\right) & =P_{1}^{+} S^{+} \operatorname{ker}\left(D+M^{(\nu+\lambda) i e_{1}}\right) \oplus P_{1}^{-} S^{+} \operatorname{ker}\left(D-M^{(\nu+\lambda) i e_{1}}\right) \\
& \oplus P_{1}^{+} S^{-} \operatorname{ker}\left(D+M^{(\nu-\lambda) i e_{1}}\right) \oplus P_{1}^{-} S^{-} \operatorname{ker}\left(D-M^{(\nu-\lambda) i e_{1}}\right),
\end{aligned}
$$

where ker means the set of null-solutions in a domain of interest $\Omega \subset \mathbb{R}^{3}$.

In this way equation (5) and hence the Dirac equation with pseudoscalar potential reduce to four equations of the form (1). 


\subsection{Force-free magnetic fields}

Force-free magnetic fields appear as an important class of special solutions of nonlinear equations of magnetohydrodynamics and are intensively studied in different branches of modern physics (see, e.g., [1], [6, [8, [10, 11], 20], 21], 22], 25], 26]). They are characterized by the following pair of equations

$$
\operatorname{div} \mathbf{B}=0
$$

and

$$
\operatorname{rot} \mathbf{B}+\nu \mathbf{B}=0,
$$

where $\nu$ is a scalar function. This system can be rewritten in the form

$$
(D+\nu) \mathbf{B}=0 .
$$

It will be more convenient for us to extend the class of solutions of (15) and to consider not only its purely vectorial solutions but more general complete biquaternionic functions. Thus we consider the equation

$$
(D+\nu) f=0,
$$

where $f$ is an $\mathbb{H}(\mathbb{C})$-valued function. Solutions of (13), (14) represent a subset of solutions of (16) which fulfil the additional requirement $\operatorname{Sc} f=0$.

Now by analogy with the preceding subsection we obtain that $f$ is a solution of (16) if and only if the functions $f^{+}=P_{1}^{+} f$ and $f^{-}=P_{1}^{-} f$ are solutions of the equations $\left(D+M^{i \nu e_{1}}\right) f^{+}=0$ and $\left(D-M^{i \nu e_{1}}\right) f^{-}=0$ respectively.

Thus the system (13), (14) reduces to a pair of equations of the form (1).

\subsection{Maxwell's equations for slowly changing media}

Consider first the general Maxwell system

$$
\begin{gathered}
\operatorname{rot} \mathbf{H}=\varepsilon \partial_{t} \mathbf{E}+\mathbf{j}, \\
\operatorname{rot} \mathbf{E}=-\mu \partial_{t} \mathbf{H}, \\
\operatorname{div}(\varepsilon \mathbf{E})=\rho, \\
\operatorname{div}(\mu \mathbf{H})=0,
\end{gathered}
$$


where $\varepsilon$ and $\mu$ are assumed to be functions of spatial coordinates only. It can be rewritten in the following form [16], 17]

$$
\left(D+M^{\vec{\varepsilon}}\right) \vec{E}=-\frac{1}{c} \partial_{t} \vec{H}-\frac{\rho}{\sqrt{\varepsilon}},
$$

and

$$
\left(D+M^{\vec{\mu}}\right) \vec{H}=\frac{1}{c} \partial_{t} \vec{E}+\sqrt{\mu} \mathbf{j},
$$

where $\vec{E}=\sqrt{\varepsilon} \mathbf{E}, \vec{H}=\sqrt{\mu} \mathbf{H}, c=1 / \sqrt{\varepsilon \mu}, \vec{\varepsilon}=\frac{\operatorname{grad} \sqrt{\varepsilon}}{\sqrt{\varepsilon}}$ and $\vec{\mu}=\frac{\operatorname{grad} \sqrt{\mu}}{\sqrt{\mu}}$.

In a sourceless time-harmonic situation we obtain the equations

$$
D_{\vec{\varepsilon}} \vec{E}=i \nu \vec{H} \quad \text { and } \quad D_{\vec{\mu}} \vec{H}=-i \nu \vec{E} .
$$

Here $\nu=\omega / c$.

The medium is said to be slowly changing when its properties change appreciably over distances much greater than the wavelength [2, 24]. Usually this is associated with the possibility of reducing the Maxwell equations (23) to the Helmholtz equations

$$
\left(\Delta+\nu^{2}\right) \vec{E}=0 \quad \text { and } \quad\left(\Delta+\nu^{2}\right) \vec{H}=0 .
$$

It is easy to check that such a reduction is possible if only $|\vec{\varepsilon}|$ and $|\vec{\mu}|$ are considered as relatively very small and the terms containing the vectors $\vec{\varepsilon}$ and $\vec{\mu}$ are supposed to be negligible. Then (23) take the form

$$
D \vec{E}=i \nu \vec{H} \quad \text { and } \quad D \vec{H}=-i \nu \vec{E}
$$

and can be diagonalized. For the functions $\vec{\varphi}=\vec{E}+i \vec{H}$ and $\vec{\psi}=\vec{E}-i \vec{H}$ we obtain the equations

$$
(D-\nu) \vec{\varphi}=0 \quad \text { and } \quad(D+\nu) \vec{\psi}=0 .
$$

Now, by analogy with equation (15) each of these two equations can be rewritten in the form (11).

\subsection{The static Maxwell system}

When the vectors of the electromagnetic field do not depend on time, from (21) and (22) we obtain two independent equations

$$
\left(D+M^{\vec{\varepsilon}}\right) \vec{E}=-\frac{\rho}{\sqrt{\varepsilon}},
$$

and

$$
\left(D+M^{\vec{\mu}}\right) \vec{H}=\sqrt{\mu} \mathbf{j} .
$$

In a sourceless situation both equations reduce to (11). 


\subsection{Two important cases}

Let us resume the results presented in this section. We see that the considered here six physical models reduce to equation (11). In the case of the static Maxwell system $\vec{\alpha}$ is a gradient of some scalar function. In all other cases in general $\vec{\alpha}$ must not be necessarily a gradient, though such a possibility is not excluded. In the first five models only one of the components of $\vec{\alpha}$ is a function while the other two are constants. Thus we are interested basically in the following two situations.

1. $\vec{\alpha}$ is a gradient of some scalar function $\varphi: \vec{\alpha}=\nabla \varphi$.

2. $\vec{\alpha}$ has the form $\vec{\alpha}=\alpha_{1}\left(x_{1}, x_{2}, x_{3}\right) e_{1}+\alpha_{2} e_{2}+\alpha_{3} e_{3}$, where $\alpha_{1}$ is a complex valued function and $\alpha_{2}, \alpha_{3}$ are complex constants (of course, when $\alpha_{1}=\alpha_{1}\left(x_{1}\right)$ we have the first case again).

\section{Factorization of the Schrödinger operator}

\subsection{Scalar Schrödinger equations}

Consider the Schrödinger operator $-\Delta+v$ applied to a scalar function $\phi$. Here $v$ is some complex valued function. Let $\vec{\alpha}$ be a purely vectorial biquaternion valued function such that

$$
D \vec{\alpha}+(\vec{\alpha})^{2}=-v
$$

Then as was observed in [3] and [4], the following equality is valid

$$
(-\Delta+v) \phi=\left(D+M^{\vec{\alpha}}\right)\left(D-M^{\vec{\alpha}}\right) \phi .
$$

This equality gives a certain relation between solutions of (11) and nullsolutions of the Schrödinger operator. Moreover, a fundamental solution of the operator $D_{\vec{\alpha}}$ can be obtained if a fundamental solution of the Schrödinger operator is given. In some cases, for instance when $\vec{\alpha}=\alpha_{1}\left(x_{1}\right) e_{1}$, this leads to construction of integral representations for solutions of (10) (see [18]).

Equation (26) represents a generalization of the famous Riccati differential equation. In [12] (see also [17]) it was shown that it is not merely a formal resemblance. On the contrary, the well known Euler theorems, the Weyr theorem as well as the Picard theorem descibing the unique properties of the Riccati equation were generalized for equation (26).

Equation (26) necessarily implies that $\vec{\alpha}$ is a gradient, because from the vector part of (26) we have that rot $\vec{\alpha}=0$. Thus it can be useful only for the first situation from Subsection 3.7. 
Let us consider the product of the operators $D_{\vec{\alpha}}$ and $D_{-} \vec{\alpha}$ in application to a twice differentiable biquaternion valued function $u$ and for any differentiable purely vectorial biquaternion $\vec{\alpha}$. We have

$$
\begin{aligned}
D_{\vec{\alpha}} D_{-\rightarrow} u & =\left(D+M^{\vec{\alpha}}\right)\left(D-M^{\vec{\alpha}}\right) \sum_{k=0}^{3} u_{k} e_{k} \\
& =\sum_{k=0}^{3}\left(M^{e_{k}}\left(D+M^{\vec{\alpha}^{(k)}}\right)\left(D-M^{\vec{\alpha}^{(k)}}\right) u_{k}\right) .
\end{aligned}
$$

For each component $u_{k}$ we use (27) and note that $\left(\vec{\alpha}^{(k)}\right)^{2}=\vec{\alpha}^{2}$. We obtain

$$
D_{\vec{\alpha}} D_{-\vec{\alpha}} u=\sum_{k=0}^{3}\left(-\Delta u_{k}-\vec{\alpha}^{2} u_{k}-\left(D \vec{\alpha}^{(k)}\right) u_{k}\right) e_{k} .
$$

From (28) we see that the equation $D_{\vec{\alpha}} D_{-\vec{\alpha}} u=0$ is equivalent to four scalar Schrödinger equations if only $D \vec{\alpha}^{(k)}$ is scalar for any $k=0,1,2,3$. It is easy to verify that it is possible if only $\vec{\alpha}$ has the following form

$$
\vec{\alpha}=\alpha_{1}\left(x_{1}\right) e_{1}+\alpha_{2}\left(x_{2}\right) e_{2}+\alpha_{3}\left(x_{3}\right) e_{3} .
$$

Let us consider this case in detail. First of all we notice that for the Dirac operator with scalar, electric or pseudoscalar potential this restriction on $\vec{\alpha}$ implies that the potential is an arbitrary function of one spatial coordinate. In the case of force-free magnetic fields (Subsection 3.4) the proportionality factor $\nu$ is a function of one variable. In the case of the electromagnetic field in a slowly changing medium (Subsection 3.5) the wave number $\nu$ is a function of one variable. Such media are known as stratified media.

For the static Maxwell system $\operatorname{div}(\varepsilon \mathbf{E})=0$, and $\operatorname{rot} \mathbf{E}=0$ we obtain that it is equivalent to (11) with $\vec{\alpha}$ having the form (29) iff the permittivity $\varepsilon$ has the following form $\varepsilon=\varepsilon_{1}\left(x_{1}\right) \varepsilon_{2}\left(x_{2}\right) \varepsilon_{3}\left(x_{3}\right)$, where $\varepsilon_{1}, \varepsilon_{2}$ and $\varepsilon_{3}$ are arbitrary functions. $\alpha_{k}$ are related to $\varepsilon_{k}$ in the following way $\alpha_{k}=\left(\partial_{k} \varepsilon_{k}\right) /\left(2 \varepsilon_{k}\right)$, $k=1,2,3$.

Thus for all considered physical models $\vec{\alpha}$ in the form (29) corresponds to quite interesting, nontrivial situations. Let us study in detail the structure of solutions of equation (10) when $\vec{\alpha}$ has the form (29).

Denote

$$
v_{k}=-D \vec{\alpha}^{(k)}-\vec{\alpha}^{2},
$$

and

$$
w_{k}=D \vec{\alpha}^{(k)}-\vec{\alpha}^{2}, \quad k=0,1,2,3
$$


Proposition 2 Let $f$ be a solution of (1) with $\vec{\alpha}$ having the form (29). Then the components $f_{k}$ are solutions of the Schrödinger equations

$$
\left(-\Delta+w_{k}\right) f_{k}=0, \quad k=0,1,2,3 .
$$

Proof. Assume that $f$ is a solution of (11). Then considering the equation $D_{-\vec{\alpha}} D_{\vec{\alpha}} f=0$ we arrive at the equations (32) for the components $f_{k}$.

The following statement gives us a method for constructing exact solutions of (11) having obtained solutions of the corresponding Schrödinger equations.

Proposition 3 Let $\vec{\alpha}$ be of the form (29) and four scalar functions $g_{k}$, $k=0,1,2,3$ satisfy the following equations

$$
\left(-\Delta+v_{k}\right) g_{k}=0
$$

Then the function

$$
f=\left(D-M^{\vec{\alpha}}\right) g
$$

is a solution of (1), where $g=\sum_{k=0}^{3} g_{k} e_{k}$.

Proof. This is an immediate consequence of (28) .

Example 4 Let $\vec{\alpha}=\sum_{k=1}^{3} \frac{1}{x_{k}-b_{k}} e_{k}$, where $b_{k}$ are arbitrary complex constants. We have

$$
v_{0}=-D \vec{\alpha}-\vec{\alpha}^{2}=0 .
$$

This means that taking any scalar harmonic function $g_{0}$ we are able to construct a solution of (11) in the form $f=\left(D-M^{\vec{\alpha}}\right) g_{0}$. Calculating $v_{1}, v_{2}$ and $v_{3}$ according to (30) we obtain

$$
\begin{aligned}
& v_{1}=2\left(\frac{1}{\left(x_{2}-b_{2}\right)^{2}}+\frac{1}{\left(x_{3}-b_{3}\right)^{2}}\right), \\
& v_{2}=2\left(\frac{1}{\left(x_{1}-b_{1}\right)^{2}}+\frac{1}{\left(x_{3}-b_{3}\right)^{2}}\right), \\
& v_{3}=2\left(\frac{1}{\left(x_{1}-b_{1}\right)^{2}}+\frac{1}{\left(x_{2}-b_{2}\right)^{2}}\right) .
\end{aligned}
$$


We will not try to find general solutions of the Schrödinger equations with these potentials. We show instead how one can always obtain a class of exact solutions of (11) and of the corresponding Schrödinger equations (33) when $\vec{\alpha}$ has the form (29).

Let us look for a one-component solution of (10): $f=f_{k} e_{k}$. We have $D f_{k} e_{k}+f_{k} \vec{\alpha}^{(k)} e_{k}=0$. That is

$$
\frac{\nabla f_{k}}{f_{k}}=-\vec{\alpha}^{(k)}
$$

It is easy to see that for $\vec{\alpha}$ of the form (29) the functions $f_{k}$ are

$f_{0}=e^{-\left(\Lambda_{1}+\Lambda_{2}+\Lambda_{3}\right)}, \quad f_{1}=e^{-\Lambda_{1}+\Lambda_{2}+\Lambda_{3}}, \quad f_{2}=e^{\Lambda_{1}-\Lambda_{2}+\Lambda_{3}}, \quad f_{3}=e^{\Lambda_{1}+\Lambda_{2}-\Lambda_{3}}$,

where $\Lambda_{k}=\Lambda_{k}\left(x_{k}\right), k=1,2,3$ is an antiderivative of $\alpha_{k}$. Thus the function $\sum_{k=0}^{3} c_{k} f_{k} e_{k}$ is a solution of (11), where $c_{k}$ are arbitrary complex constants.

Concerning the Schrödinger equations (33) it is useful to consider the quaternionic Riccati equation (26). Any solution of it can be represented in the form $\vec{\alpha}=\frac{\nabla \varphi}{\varphi}$, where $\varphi$ is a solution of the equation $-\Delta \varphi+v \varphi=0$ (see [12], [17]). For $\vec{\alpha}^{(k)}$ from (39) we obtain that $\vec{\alpha}^{(k)}=\frac{\nabla \varphi_{k}}{\varphi_{k}}$, where $\varphi_{k}=1 / f_{k}$ and consequently the functions $\varphi_{k}$ are solutions of (33) for a corresponding $k$.

Let us see what are the functions $f_{k}$ and $\varphi_{k}$ in Example 4

Example 5 For $\vec{\alpha}$ from the preceding example we have

$$
\begin{gathered}
f_{0}=\frac{1}{\left(x_{1}-b_{1}\right)\left(x_{2}-b_{2}\right)\left(x_{3}-b_{3}\right)}, \quad f_{1}=\frac{\left(x_{2}-b_{2}\right)\left(x_{3}-b_{3}\right)}{x_{1}-b_{1}}, \\
f_{2}=\frac{\left(x_{1}-b_{1}\right)\left(x_{3}-b_{3}\right)}{x_{2}-b_{2}}, \quad f_{3}=\frac{\left(x_{1}-b_{1}\right)\left(x_{2}-b_{2}\right)}{x_{3}-b_{3}} .
\end{gathered}
$$

Hence the function $f=\sum_{k=0}^{3} c_{k} f_{k} e_{k}$ is a solution of (1) and the functions

$$
\begin{gathered}
\varphi_{0}=\left(x_{1}-b_{1}\right)\left(x_{2}-b_{2}\right)\left(x_{3}-b_{3}\right), \quad \varphi_{1}=\frac{x_{1}-b_{1}}{\left(x_{2}-b_{2}\right)\left(x_{3}-b_{3}\right)}, \\
\varphi_{2}=\frac{x_{2}-b_{2}}{\left(x_{1}-b_{1}\right)\left(x_{3}-b_{3}\right)}, \quad \varphi_{3}=\frac{x_{3}-b_{3}}{\left(x_{1}-b_{1}\right)\left(x_{2}-b_{2}\right)}
\end{gathered}
$$

are solutions of (33) with potentials (35)-(38) respectively.

Proposition 6 Let $\vec{\alpha}$ be of the form (29) and $f=\left(D-M^{\vec{\alpha}}\right) g$ a solution of (11), where $g=\sum_{k=0}^{3} g_{k} e_{k}$. Then necessarily $g_{k}$ are solutions of (33) respectively. 
Proof. Consider

$$
\begin{aligned}
\left(D+M^{\vec{\alpha}}\right) f & =\left(D+M^{\vec{\alpha}}\right)\left(D-M^{\vec{\alpha}}\right) \sum_{k=0}^{3} g_{k} e_{k} \\
& =\sum_{k=0}^{3}\left(M^{e_{k}}\left(D+M^{\vec{\alpha}^{(k)}}\right)\left(D-M^{\vec{\alpha}^{(k)}}\right) g_{k}\right) \\
& =\sum_{k=0}^{3}\left(M^{e_{k}}\left(-\Delta+v_{k}\right) g_{k}\right) .
\end{aligned}
$$

From assumption of the proposition we obtain that $\left(-\Delta+v_{k}\right) g_{k}, k=$ $0,1,2,3$.

Let $\Omega$ be a domain in $\mathbb{R}^{3}$ which in particular may coincide with the whole space. Let $F(\Omega)$ and $G(\Omega)$ be some functional spaces. The $\mathbb{H}(\mathbb{C})$-valued function $f$ is said to belong to a functional space if each of its components $f_{k}$ belongs to it.

Proposition 7 Let $\vec{\alpha}$ be of the form (29). Assume that the equation $(-\Delta+$ $\left.w_{k}(\mathbf{x})\right) u(\mathbf{x})=\mu(\mathbf{x}), \mathbf{x} \in \Omega, k=0,1,2,3$ is solvable for any $\mu \in F(\Omega)$ and the solution $u$ belongs to $G(\Omega)$. Then the equation

$$
\left(D-M^{\vec{\alpha}}\right) g=f
$$

is solvable for any $f \in F(\Omega)$ and the solution $g$ belongs to $\operatorname{im} D_{\vec{\alpha}}(G(\Omega))$.

Proof. Let $u_{k}, k=0,1,2,3$ be solutions of the equations $\left(-\Delta+w_{k}\right) u_{k}=$ $f_{k}$. Then $g=\left(D+M^{\vec{\alpha}}\right) \sum_{k=0}^{3} u_{k} e_{k}$ is a solution of (40).

Remark 8 We do not specify the functional spaces here because the results on solvability of the inhomogeneous Schrödinger equation are numerous and correspond to very different situations. Let us give an example.

Example 9 Let $\Omega=\mathbb{R}^{3}$ and $w$ has the form

$$
w=\widehat{w}-m^{2},
$$

where $\widehat{w} \in C_{0}^{\infty}\left(\mathbb{R}^{3}\right), m>0$. The equation $(-\Delta+w) u=\mu$ is uniquely solvable [7] for any $\mu \in L_{2, a}$ and $u \in H_{l o c}^{2}\left(\mathbb{R}^{3}\right), u=O\left(\frac{1}{|\mathbf{x}|}\right), \frac{\partial u}{\partial|\mathbf{x}|}-i m u=o\left(\frac{1}{|\mathbf{x}|}\right)$ for $|\mathbf{x}| \rightarrow \infty$. Here $L_{2, a}$ denotes the space of square integrable functions with support in a ball of radius a.

Thus from Proposition 7 we have the solvability of $\sqrt{40}$ for any $\vec{\alpha}$ such that $w_{k}$ defined by (31) have the form (41), for example, for $\vec{\alpha}=$ $\sum_{k=1}^{3} \beta_{k}\left(x_{k}\right)+i m_{k}$, where $\beta_{k} \in C_{0}^{\infty}\left(\mathbb{R}^{3}\right)$ and $m_{k}>0$. 
Proposition 10 Under the conditions of Proposition 7 any solution of (1) from $F(\Omega)$ has the form

$$
f=\left(D-M^{\vec{\alpha}}\right) g
$$

where $g=\sum_{k=0}^{3} g_{k} e_{k}$. and $g_{k}$ satisfy the equations (33) in $\Omega$.

Proof. From Proposition 7 it follows that $f$ can be represented in the form (42), where $g \in \operatorname{im} D_{\vec{\alpha}}(G(\Omega))$. From Proposition [6] we obtain that $g_{k}$ are solutions of (33).

For the Schrödinger operator there are developed different approaches for obtaining asymptotic fundamental solutions under some additional assumptions. A fundamental solution can be used for construction of a right-inverse operator for the Schrödinger operator which gives a possibility to solve inhomogeneous equations. In the following proposition we show that having constructed right-inverse operators for the Schrödinger operators $-\Delta+v_{k}$, $k=0,1,2,3$, one can construct a right-inverse operator for $D_{\vec{\alpha}}$.

Proposition 11 Let $\vec{\alpha}$ be of the form (29) and $T_{k}$ such operators that for any $\varphi \in F(\Omega):\left(-\Delta+v_{k}\right) T_{k} \varphi=\varphi$ in $\Omega, k=0,1,2,3$. Then for any $f=\sum_{k=0}^{3} f_{k} e_{k} \in F(\Omega)$ we have $D_{\vec{\alpha}} T_{\vec{\alpha}} f=f$ in $\Omega$, where $T_{\vec{\alpha}} f=$ $\left(D-M^{\vec{\alpha}}\right)\left(\sum_{k=0}^{3}\left(T_{k} f_{k}\right) e_{k}\right)$.

Proof. Consider

$$
\begin{aligned}
\left(D+M^{\vec{\alpha}}\right) T_{\vec{\alpha}} f & =\sum_{k=0}^{3}\left(M^{e_{k}}\left(D+M^{\vec{\alpha}^{(k)}}\right)\left(D-M^{\vec{\alpha}^{(k)}}\right)\left(T_{k} f_{k}\right)\right) \\
& =\sum_{k=0}^{3}\left(M^{e_{k}}\left(-\Delta+v_{k}\right) T_{k} f_{k}\right)=\sum_{k=0}^{3} f_{k} e_{k}=f .
\end{aligned}
$$

\subsection{Schrödinger equations with quaternionic potentials}

Let us assume that $\vec{\alpha}$ has the form

$$
\vec{\alpha}=\alpha_{1}\left(x_{1}, x_{2}, x_{3}\right) e_{1}+\alpha_{2} e_{2}+\alpha_{3} e_{3}
$$

where $\alpha_{1}$ is an arbitrary complex valued differentiable function and $\alpha_{2}, \alpha_{3}$ are complex constants. In Section 3 we saw that the first five physical models considered here correspond to this situation. 
Note that in this case $D \vec{\alpha}=D \vec{\alpha}^{(1)}=-D \vec{\alpha}^{(2)}=-D \vec{\alpha}^{(3)}$. Hence from (28) we obtain

$$
D_{\vec{\alpha}} D_{-\rightarrow} u=-\Delta u-\vec{\alpha}^{2} u-(D \vec{\alpha})\left(u_{0} e_{0}+u_{1} e_{1}-u_{2} e_{2}-u_{3} e_{3}\right),
$$

where $u=\sum_{k=0}^{3} u_{k} e_{k}$.

Denote $C u=-e_{1} u e_{1}=u_{0} e_{0}+u_{1} e_{1}-u_{2} e_{2}-u_{3} e_{3}, A u=-\Delta u-\vec{\alpha}^{2} u$ and $B u=-(D \vec{\alpha}) u$. That is

$$
D_{\vec{\alpha}} D_{-\vec{\alpha}}=A+B C
$$

and

$$
D_{-\rightarrow} D_{\vec{\alpha}}=A-B C .
$$

Denote $Q^{ \pm}=\frac{1}{2}\left(I \pm i e_{1} C\right)$.

Proposition 12 Solutions of equation (11) (with $\vec{\alpha}$ of the form (43)) have the form $f=D_{-} \vec{\alpha} u$, where $u=Q^{+} v+Q^{-} w$ and $v, w$ are solutions of the following Schrödinger equations with quaternionic potentials

$$
\left(A+B i e_{1}\right) v=0
$$

and

$$
\left(A-B i e_{1}\right) w=0
$$

respectively.

Proof. First, we notice that $Q^{ \pm} B=B Q^{ \pm}$and $Q^{ \pm} C=\frac{1}{2}\left(C \pm i e_{1}\right)=$ $\frac{1}{2}\left(C_{i e} \pm I\right) i e_{1}= \pm Q^{ \pm} i e_{1}$.

Applying $Q^{+}$and $Q^{-}$to the equation

$$
(A+B C) u=0
$$

we see that it is equivalent to the pair of equations $\left(A+B i e_{1}\right) Q^{+} u=0$, $\left(A-B i e_{1}\right) Q^{-} u=0$, and $Q^{+}, Q^{-}$commute with the operators in parentheses and represent a pair of mutually complementary projection operators on the space of $\mathbb{H}(\mathbb{C})$-valued functions. Thus we have that $u$ is a solution of (47) iff $u=Q^{+} v+Q^{-} w$ and $v, w$ are solutions of (45) and (46) respectively. Now using (44) we finish the proof.

Thus solution of (11) with $\vec{\alpha}$ of the form (43) reduces to solution of the Schrödinger equations with quaternionic potentials (45) and (46) which can be rewritten in a more explicit form as follows

$$
\left(-\Delta-\left(\vec{\alpha}^{2}-i D \alpha_{1}\right) I\right) v=0
$$

and

$$
\left(-\Delta-\left(\vec{\alpha}^{2}+i D \alpha_{1}\right) I\right) w=0
$$


Remark 13 In the case when $\alpha_{1}$ does not depend on $x_{1}: \alpha_{1}=\alpha_{1}\left(x_{2}, x_{3}\right)$ it is easy to see that equations (48) and (49) are not independent. We have that if $v$ is a solution of (48) then $w=i e_{1} v$ is a solution of (49) and vice versa. Using this fact we obtain a one-to-one correspondence between solutions of (47) and solutions of (48). Such a correspondence is given by the operator

$$
\Pi=\frac{1}{2}\left(I+i e_{1}-C+i e_{1} C\right) .
$$

It can be verified immediately that $\Pi^{2}=I$ and that $u$ is a solution of (47) if and only if $v=\Pi u$ is a solution of (48) and vice versa. That is when $\vec{\alpha}=\alpha_{1}\left(x_{2}, x_{3}\right) e_{1}+\alpha_{2} e_{2}+\alpha_{3} e_{3}$ where $\alpha_{2}, \alpha_{3}$ are constants, solution of (1) reduces to solution of one Schrödinger equation with quaternionic potential 48).

Remark 14 Some classes of solutions of (48) and (49) can be obtained reducing the equations to scalar Schrödinger's equations using the following idea (proposed in [15] in another setting). Consider equation [48). If $\left(i D \alpha_{1}-\vec{\alpha}^{2}\right) \in \mathfrak{S}$ or $D \alpha_{1} \in \mathfrak{S}$ then we can look for solutions of (48) in the form $v=\left(i D \alpha_{1}+\vec{\alpha}^{2}\right) f$ or $v=\left(D \alpha_{1}\right) f$ respectively where $f$ is an unknown function. In the first case the equation reduces to the Laplace equation $\Delta v=0$ and in the second to the Schrödinger equation $\left(\Delta+\vec{\alpha}^{2}\right) v=0$.

Suppose that neither of these is the case. Then denote $\vec{\beta}=i D \alpha_{1}$ and introduce $\beta_{0}$ as a scalar square root of $\vec{\beta}^{2}$. The complex quaternions $\beta=$ $\beta_{0}+\vec{\beta}$ and $\bar{\beta}=\beta_{0}-\vec{\beta}$ are conjugate zero divisors. Equation (48) then can be rewritten as follows

$$
\left(-\Delta-\left(\beta_{0}+\vec{\alpha}^{2}\right)+\beta\right) v=0 .
$$

Looking for its solutions of the form $v=\bar{\beta} f$ we reduce it to the scalar Schrödinger equation $\left(\Delta+\left(\beta_{0}+\vec{\alpha}^{2}\right)\right) v=0$.

\section{Conclusions}

We have shown that an ample class of physically meaningful partial differential systems of first order are equivalent to a single quaternionic equation which in its turn reduces in general to a Schrödinger equation with quaternionic potential, and in some situations considered in Subsection 4.1 this last can be diagonalized. The rich variety of methods developed for different problems corresponding to the Schrödinger equation can be applied to the systems considered in the present work. 


\section{References}

[1] Athanasiadis C, Costakis G and Stratis I G On some properties of Beltrami fields in chiral media. Reports on Mathematical Physics 45 (2000), 257-271.

[2] Babich V M and Buldyrev V S Short-Wavelength Diffraction Theory. Asymptotic Methods (Springer, Berlin, 1991).

[3] Bernstein S Factorization of solutions of the Schrödinger equation, In: Proceedings of the symposium Analytical and numerical methods in quaternionic and Clifford analysis, Seiffen, 1996.

[4] Bernstein S and Gürlebeck K On a higher dimensional Miura transform, Complex Variables, 1999, vol.38, 307-319.

[5] Bjorken J and Drell S Relativistic quantum mechanics. The McGraw-Hill Companies, Inc, 1998.

[6] Clegg J R, Browning P K, Laurence P, Bromage B J I and Stredulinsky E On the representation of inhomogeneous linear force-free fields. Journal of Mathematical Physics, 2000, vol. 41, 6783-6807.

[7] Egorov $\mathrm{Yu} \mathrm{V}$ and Shubin M A Foundations of the Classical Theory of Partial Differential Equations (Encyclopaedia of Mathematical Sciences, 30). Springer Verlag, 1998.

[8] Feng Qingzeng On force-free magnetic fields and Beltrami flows. Applied Mathematics and Mechanics (English Edition) 18, 997-1003 (1997)

[9] Gürlebeck K and Sprössig W Quaternionic and Clifford Calculus for Physicists and Engineers. John Wiley \& Sons, 1997.

[10] Kaiser R, Neudert M and von Wahl W On the existence of force-free magnetic fields with small nonconstant $\alpha$ in exterior domains. Communications in Mathematical Physics 211, 111-136 (2000)

[11] Kamien R. D. Force free configurations of vortices in high temperature superconductors near the melting transition. arXiv:cond-mat/9804287 v3, 10 pp. (1998).

[12] Kravchenko V G, Kravchenko V V and Williams B D A quaternionic generalization of the Riccati differential equation. Kluwer Acad. Publ., "Clifford Analysis and Its Applications", Ed. by F. Brackx et al., 143$154,2001$. 
[13] Kravchenko V V On a biquaternionic bag model. Zeitschrift für Analysis und ihre Anwendungen, 1995, v. 14, \# 1, 3-14.

[14] Kravchenko V V Exact solutions of the Dirac equation with harmonic pseudoscalar, scalar or electric potential. Journal of Physics A., 1998, v. 31, \# 37, 7561-7575.

[15] Kravchenko V V On a new approach for solving Dirac equations with some potentials and Maxwell's system in inhomogeneous media. Birkhäuser Verlag, Operator Theory: Advances and Applications, vol. 121, 278-306, 2001.

[16] Kravchenko V V Quaternionic reformulation of Maxwell's equations for inhomogeneous media and new solutions. Zeitschrift für Analysis und ihre Anwendungen 21, 21-26 (2002)

[17] Kravchenko V V Applied quaternionic analysis. Heldermann-Verlag, Research and Exposition in Mathematics Series, v. 28, 2003.

[18] Kravchenko V V On Beltrami fields with nonconstant proportionality factor. Journal of Physics A, 2003, v. 36, 1515-1522.

[19] Kravchenko V V and Shapiro M V Integral representations for spatial models of mathematical physics. Addison Wesley Longman Ltd., Pitman Res. Notes in Math. Series, v. 351, 1996.

[20] Lifschitz A Magnetohydrodynamics and spectral theory. Dordrecht: Kluwer Acad. Publ., 1989

[21] Priest E and Hood A (eds.) Advances in solar system magnetohydrodynamics. Cambridge: Cambridge University Press, 1991

[22] Schnack D D, Mikic Z, Barnes D C and Van Hoven G Magnetohydrodynamic simulation of coronal magnetic fields. Computer Physics Communications 59, 21-37 (1990)

[23] Thaller B The Dirac equation. Springer-Verlag, 1992.

[24] Vinogradova M B, Rudenko O V and Sukhorukov A P Theory of waves. Moscow, Nauka, 1990 (in Russian).

[25] Yoshida Z Applications of Beltrami functions in plasma physics. Nonlinear Analysis, Theory \& Applications 30, 3617-3627 (1997) 
[26] Zaghloul H and Barajas O Force-free magnetic fields. American Journal of Physics 58, 783-788 (1990) 